\title{
Characterization of DNA nanostructure stability by size exclusion chromatography
}

\author{
Nicole I. Langlois ${ }^{\dagger}$ and Heather A. Clark $*{ }^{\dagger *}$ \\ 'Department of Chemistry and Chemical Biology, Northeastern University, Boston, MA, United States \\ tDepartment of Bioengineering, Northeastern University, Boston, MA, United States
}

KEYWORDS: DNA nanotechnology, stability analysis, SEC-HPLC

\begin{abstract}
DNA-based nanostructures (DNs) are advantageous for the design of functional materials for biology and medicine due to the nanoscale control provided by their predictable self-assembly. However, the use of DNs in vivo has been limited due to structural instability in biofluids. As the stability of a particular DN sets the scope of its potential biological applications, efficient methods to characterize stability are required. Here, we apply size exclusion chromatography (SEC) to study the stability of a tetrahedron DNA nanostructure (TDN) and demonstrate the analytical capabilities of our method in characterizing degradation by enzymes and a diluted human serum matrix. We show that SEC analysis can reliably assay TDN degradation by a nuclease through direct injection and peak integration. Furthermore, data analysis using a ratio chromatogram technique enables TDN peak deconvolution from the matrix of serum proteins. Using our method, we found that TDNs exhibit half-lives of 23.9 hours and 10.1 hours in $20 \%$ and $50 \%$ diluted human serum, respectively, which is consistent with reported stability studies in $10 \%$ fetal bovine serum. We anticipate that this method can be broadly applicable to characterize a variety of DNs and serve as an efficient technique toward analysis of the stability of new DN designs in complex biological matrixes.
\end{abstract}

\section{INTRODUCTION}

Comprehensive assessment of the stability of DNA nanostructures (DNs) is essential to understanding the conditions under which the assemblies remain intact and to predicting suitability for use in various biological applications. Thus, several techniques have been adopted for understanding the structural or biological stability of $\mathrm{DNs}^{1-6}$, most notably gel electrophoresis. As the field of DNA nanotechnology has progressed rapidly from simple structural motifs toward highly complex and functionalized nanoarchitectures designed for in vivo use, there are additional challenges posed to existing analysis methods. For example, when spiked into biological media (e.g. serum, urine, saliva, etc.), DNs face two primary modes of destabilization: (1) ionic, from the displacement of divalent magnesium ions required to form the complexes, and (2) enzymatic, from rapid digestion by nucleases present in the biofluid. Gel electrophoresis is useful for monitoring these routes of degradation as it is simple to adopt by most laboratories for examining the impact of individual components (e.g. low-Mg buffers, urea, specific enzymes) on the stability of DNs ${ }^{1}$. However, for analysis in complex biofluids, high background signal from biological proteins requires that the DNA is extracted from the test matrix prior to gel analysis; the resulting data typically probes DN stability using denatured structures ${ }^{7}$. Fluorescence and FRET-based methods can also assess the kinetics of degradation of DNs over time, but they require incorporation of covalently conjugated fluorophores or intercalating dyes, which can potentially be skewed by background signal from labeled degradation products ${ }^{8}$. Molecular imaging techniques including AFM and TEM imaging can provide a detailed insight into the substructure-specific degradation of DNs, although these methods face significant barriers to wide-spread adoption including specialized training and instrument access as well as laborious data analysis.

There is a need to develop robust analytical techniques ${ }^{9}$ to characterize DN stability under diverse biological conditions as well as methods for standardized analysis and reporting of stability data. While it is well accepted that DNs are more resistant to nuclease degradation than their duplex counterparts, it is challenging to otherwise compare results across studies ${ }^{7}$. Many DN analyses apply different characterization methods (e.g. gels, AFM, fluorescence) to describe different structures (e.g. 2Dorigami, wireframes, TDNs, etc.) by different metrics (e.g. halflife, percent intact, lifetime). As the breadth of biological applications for DNs continue to increase, methods to efficiently compare long-term biostability between DN designs are even more important.

Size exclusion chromatography (SEC) is a form of high-performance liquid chromatography (HPLC) that enables flexible analysis of biomolecules in their native or denatured states, based on separation by effective molecular size (i.e. hydrodynamic volume) in solution ${ }^{10}$. As analytes travel through the column, the smaller components travel within the network of porous particles while larger species are excluded from the pores, resulting in an elution order of decreasing molecular size. This approach is useful for analyzing and purifying mixtures of differently sized biomolecules, with resolution largely driven by the sample interactions with stationary phase particles and the column dimensions (i.e. column fractionation range). SECbased methods are the gold standard for biopharmaceutical 
characterization in terms of separating protein monomers from aggregates and monitoring long-term formulation stability ${ }^{11}$. In the field of DNA nanotechnology, SEC methods have been reported for purification of functionalized $\mathrm{DNs}^{12,13}$. Thus, this technique has the potential to enhance analytical characterization of the degradation profiles of DNs in biological matrixes.

Here, we have developed a method utilizing SEC for characterization of DNA nanostructure stability in human serum. We selected SEC-HPLC as our analytical technique as it enables size-based separation of biomolecules in their native configurations without requiring pre-analysis purification, a major advantage over existing methods. Additionally, use of a variable wavelength detector enables online, label-free monitoring of analytes across a spectrum of absorbance to aid in peak identification and analysis. We developed our method using a fourstrand tetrahedron DNA nanostructure (TDN) with 17-bp edges ${ }^{14}$ as our test structure, as it represents one of the most common DN designs used in biosensing and biomedical applications ${ }^{15}$. This particular TDN has been well-characterized in the literature ${ }^{16,17}$, providing a baseline with which we could compare our results. We demonstrate that the enzymatic breakdown of a TDN by nucleases over specific time intervals can be resolved using SEC-HPLC by directly injecting an incubated sample. The integrated peak area on the resulting chromatograms can be plotted as a function of time to determine the TDN half-life. Moreover, we show that for sample test conditions that are not compatible with direct HPLC injection (i.e. high protein content which would clog the system), we can employ a ratiometric analysis technique ${ }^{18}$ to extract DN stability information from a diluted human serum matrix.

\section{EXPERIMENTAL SECTION}

Materials. Custom DNA oligonucleotides were purchased from MilliporeSigma with HPLC purification and all sequences are listed in Figure S1. Trizma ${ }^{\circledR}$ (Tris) base, hydrochloric acid $(\mathrm{HCl})$, and magnesium chloride $\left(\mathrm{MgCl}_{2}\right)$ were obtained from Sigma-Aldrich. Sodium chloride $(\mathrm{NaCl})$ and phosphate buffered saline (PBS) were purchased from Fisher Scientific. Water was obtained from a Milli-Q purification system from MilliporeSigma. Aqueous mobile phases were filtered through $0.22 \mu \mathrm{m}$ polyethersulfone (PES) membrane filters (Fisher Scientific) and used within two weeks.

Instrumentation. Chromatographic analyses were performed using an Agilent 1260 Infinity II LC System, equipped with a vial sampler, quaternary pump, wide-range diode array detector, and ChemStation software. The detector online monitoring wavelengths were set at $260 \mathrm{~nm}$ and $280 \mathrm{~nm}$ for optimal analysis of the DNA and serum proteins, respectively.

Self-Assembly of DNA Tetrahedrons. Assembly of TDNs was accomplished following the original annealing protocol by Goodman et $\mathrm{al}^{14}$. Equimolar quantities $(2 \mu \mathrm{M})$ of the four TDN component strands were combined in TEM buffer (10 mM Tris, $1 \mathrm{mM}$ EDTA, $15 \mathrm{mM} \mathrm{MgCl}$, $\mathrm{pH}$ 8.00) and annealed by heating to $95^{\circ} \mathrm{C}$ for $2 \mathrm{~min}$, then cooling to $20^{\circ} \mathrm{C}$ over $2 \mathrm{~min}$ in a Mastercycler nexus GX2 thermal cycler (Eppendorf).

Purification of TDNs by SEC. A method for purification of TDNs from multimers was developed following an established approach ${ }^{12}$, using a semi-preparative SEC column (Superdex ${ }^{\circledR} 200$ Increase 10/300 GL, Cytiva, $30 \mathrm{~cm}$ x $10 \mathrm{~mm}$ ). The typical mobile phase was TN buffer $(30 \mathrm{mM}$ Tris- $\mathrm{HCl}, 450 \mathrm{mM}$ $\mathrm{NaCl}, \mathrm{pH} 7.40$ ) and the flow rate was set to $0.50 \mathrm{~mL} \mathrm{~min}^{-1}$. Other purification conditions that we evaluated are listed in
Figure S2. Samples of $500 \mu \mathrm{L}$ of annealed TDNs are injected onto the column, with corresponding TDN fractions collected manually. Collected fractions were pooled and concentrated using Amicon ${ }^{\circledR}$ Ultra- $0.5 \mathrm{~mL}$ centrifugal filters (30K MWCO, MilliporeSigma), centrifuged in intervals at $2,000 \times \mathrm{xg}\left(4^{\circ} \mathrm{C}\right) . \mathrm{Pu}-$ rified TDNs were compared by $\mathrm{A}_{260}$ values measured using a NanoDrop 2000c spectrophotometer (Thermo Fisher). Quantities were calculated using an reported extinction coefficient of $1.87\left(\mathrm{x} 10^{6} \mathrm{~L} \mathrm{~mol}^{-1} \mathrm{~cm}^{-1}\right)^{12}$. Recovery and purity of TDNs were evaluated through replicate purifications of annealed TDNs (50 $\mu \mathrm{L}, 2 \mu \mathrm{M})$. After concentration, samples were re-injected and the proportional integrated peak area was used to determine the amount of recovered TDNs and their purity.

Serum Sample Preparation. Single-donor whole human serum (HS) was obtained fresh from ZenBio Inc. (Fisher Scientific). Aliquots were prepared immediately and frozen at $-80^{\circ} \mathrm{C}$. Frozen HS aliquots were thawed on ice directly before use. All HS dilutions were prepared using PBS (10 mM phosphate, 137 $\mathrm{mM} \mathrm{NaCl}, 2.7 \mathrm{mM} \mathrm{KCl}, \mathrm{pH}$ 7.40) prior to adding in TDNs to reach the final effective $\mathrm{HS}$ concentration.

Chromatographic Analysis of TDNs and HS by SEC. A method for characterization of TDNs in HS was developed using a TSK gel SuperSW2000 column (30 cm x $4.6 \mathrm{~mm}$, Tosoh Bioscience). Mobile phase buffer conditions tested included TN, 0.3X TN (10 mM Tris- $\mathrm{HCl}, 150 \mathrm{mM} \mathrm{NaCl}, \mathrm{pH} 7.40)$, and PBS. Flow rates tested include $0.30,0.35$, and $0.40 \mathrm{~mL} \mathrm{~min}^{-1}$. Samples of 5\% HS and unpurified TDN were prepared separately and analyzed. The optimal conditions were selected based on the TDNs eluting in the lowest background of the HS sample, while aggregates can be separated from pure TDNs with a resolution (Rs) greater than 1.0. Analysis of TDN intermediates was performed by annealing component strands together in a stepwise fashion. Sequential equimolar $(2 \mu \mathrm{M})$ addition of $\mathrm{T} 1, \mathrm{~T} 1+\mathrm{T} 2, \mathrm{~T} 1+\mathrm{T} 2+\mathrm{T} 3$, and $\mathrm{T} 1+\mathrm{T} 2+\mathrm{T} 3+\mathrm{T} 4$ was performed in TEM buffer and annealed as before. Samples were simultaneously analyzed by $6 \%$ native PAGE $(1-\mu \mathrm{L}$ DNA loaded) and our analytical SEC method (10- $\mu \mathrm{L}$ injections).

Polyacrylamide Gel Electrophoresis (PAGE). Solutions of 40\% Acrylamide:bis-Acrylamide (29:1), Tris-AcetateEDTA buffer (TAE), Tetramethylethylenediamine (TEMED), and ammonium persulfate (APS) were obtained from Fisher Scientific. Polyacrylamide gels were prepared $(6 \% \mathrm{PA}, 1 \mathrm{X}$ TAE) using Novex ${ }^{\mathrm{TM}}$ Bolt mini cassettes $(1.0 \mathrm{~mm})$ according to manufacturer specifications and run on an Invitrogen ${ }^{\mathrm{TM}}$ XCell SureLock ${ }^{\mathrm{TM}}$ Mini-Cell system (Thermo Fisher) at $120 \mathrm{~V}$ on ice. Sample bands were visualized by staining the gels for $15 \mathrm{~min}$ in 1X GelRed DNA staining solution (Biotium, MilliporeSigma), then imaged using an Axygen Gel Documentation System (Corning). For protein staining, the gels were incubated for 1 hour in $1 \mathrm{X}$ GelCode $^{\mathrm{TM}}$ Blue Safe Protein Stain (Thermo Fisher), followed by overnight de-staining in water.

Analytical Calibration of SEC Method. A calibration curve for TDN samples was prepared using purified TDNs concentrated to $\sim 50 \mathrm{ng} \mu \mathrm{L}^{-1}$. Samples were prepared over the expected range of $0.5-50 \mathrm{ng}_{\mu \mathrm{L}^{-1}}$ through serial dilution in PBS. Each dilution was measured by Nanodrop $(1 \mu \mathrm{L})$ to calculate the total DNA concentration and injected on the HPLC $(10 \mu \mathrm{L})$ to determine the TDN peak area on the $\mathrm{A}_{260}$ chromatograms. Linear regression analysis was used to find the relationship between the amount of TDN injected and the resulting area. The limit of quantification (LOQ) was determined by the sum of the mean area for a blank sample $(10 \mu \mathrm{L}$ PBS $)$ over the TDN 
integration range and three times the standard deviation of our lowest concentration sample. The accuracy of the resulting linear calibration curve was determined by preparing a separate batch of purified TDNs and adjusting to three different concentrations across the calibration range. Each sample was measured by Nanodrop and HPLC analysis as before, and the error percentage was determined by comparing the two values. The intra-day precision of the method was evaluated using six replicate, $10-\mu \mathrm{L}$ injections of $5 \% \mathrm{HS}$ and TDN (20 ng $\left.\mu \mathrm{L}^{-1}\right)$, respectively. Inter-day precision was estimated through comparison of the TDN peak retention time across several weeks.

DNase Degradation Assay by Direct Injection. Purified TDNs (500 ng) were incubated with $1.56 \mathrm{U} \mathrm{mL}^{-1}$ of DNaseI (New England BioLabs) in 1X DNase reaction buffer $(50 \mu \mathrm{L})$. Samples were mixed immediately before the first timepoint, then incubated at $37^{\circ} \mathrm{C}$. In 15 -min increments, samples aliquots were brought to the HPLC autosampler to be injected.

SEC Analysis of TDN Stability in HS. Samples of TDN $(\sim 1000 \mathrm{ng})$ were mixed with HS in PBS to final concentrations of $20 \% \mathrm{HS}$ or $50 \% \mathrm{HS}$. Immediately, an aliquot was removed, diluted with PBS, and injected on the HPLC. The mixed samples were then moved to a thermal cycler to incubate at $37^{\circ} \mathrm{C}$. Aliquots were diluted four-fold for the $20 \% \mathrm{HS}$ samples and ten-fold for the $50 \%$ HS samples to bring the effective concentration to $5 \%$ HS prior to injection to prevent clogging in the HPLC system. At specified incubation timepoints, aliquots were removed, diluted, and then directly injected for analysis. Independent samples of separately incubated HS and TDN were also analyzed through this method.

Chromatographic Data Analysis. For all analyses, chromatographic data was exported from the ChemStation software and visualized and analyzed using Origin 2021b. The area-under-the-curve (AUC) for each sample was measured by peak integration on the baseline-subtracted data. Detailed information on integration analysis methods is listed in the Supporting Information. For the stability in HS study, we adapted a ratiometric analysis technique developed by Synovec and colleagues ${ }^{18,19}$ involving a "ratio chromatogram" to deconvolute the TDN peak from the co-eluting HS matrix as follows.

We can describe the chromatogram of a multi-component sample as resulting from the detected absorbance over time, $A^{\lambda_{1}}(t)$, of a sample, $S$, at wavelength $1\left(\lambda_{1}\right)$ - where $S$ contains $M$ components. Assuming the absorbance is directly proportional to concentration and independent of noise, the data can be described by Eq 1.,

$$
A^{\lambda_{1}}(t)=\sum_{j=1}^{M} \epsilon_{j, \lambda_{1}} b C_{j, S} D_{j}(t)
$$

where $\epsilon_{j, \lambda_{1}}$ is the extinction coefficient at wavelength 1 for a particular analyte, $j, b$ is the optical pathlength of the detector, $C_{j, S}$ is the injected concentration of analyte $j$ within sample $S$, and $D_{j}(t)$ is the dispersed concentration profile of analyte $j$ over time. As a unique optical absorbance spectrum is a characteristic of a pure sample, we can use the ratio of absorbance at two different wavelengths as a qualitative metric to identify a particular sample. For example, it is widely accepted that the ratio of absorbance at $260 \mathrm{~nm}$ to $280 \mathrm{~nm}\left(A_{260} / A_{280}\right)$ is a key metric for assessing the purity of DNA and protein samples. An $A_{260} / A_{280}$ value of $\sim 1.80$ is regarded as a DNA sample free of protein contamination, while an $A_{260} / A_{280}$ value of $\sim 0.57$ represents a pure protein sample (serum albumin) free of DNA. If we examine the point-by-point ratio of detector signal monitored at these two wavelengths, we can generate a ratio chromatogram, $R^{\lambda_{1} / \lambda_{2}}(t)$. For a sample with only one analyte, the elution region of the chromatogram is that of a pure component, thus the values of $b, C_{j, s}$, and $D_{j}(t)$ are identical. The ratio chromatogram can then be represented by Eq 2 .,

$$
R^{\frac{\lambda_{260}}{\lambda_{280}}}(t)_{j=1}=\frac{A^{\lambda 260}(t)}{A^{\lambda_{280}}(t)}=\frac{\epsilon_{j,} \lambda_{260}}{\epsilon_{j,} \lambda_{280}}
$$

Above a defined noise threshold, the ratio chromatogram simplifies to a plateau over the pure-elution region with an intensity consistent with the identity of the pure analyte. For a multi-component sample with co-eluting analytes, i.e. DNA in a protein mixture, $D_{j}(t)$ is not seen as identical by the detector at the two wavelengths. Thus, we arrive at Eq 3.,

$$
R^{\frac{\lambda_{260}}{\lambda_{280}}}(t)_{j=M}=\frac{A^{\lambda_{260}(t)}}{A^{\lambda_{280}(t)}}=\frac{\sum_{j=1}^{M} \epsilon_{j,} \lambda_{260} D_{j}(t)}{\sum_{j=1}^{M} \epsilon_{j,} \lambda_{280} D_{j}(t)}
$$

As in Eq 2, the ratio chromatogram simplifies to a plateau over a region of pure elution of a single component. In addition, for regions where the elution peaks of multiple analytes are overlapping, the signal ratio will deviate from a linear horizontal region with an intensity related to the change in concentration proportion at each point in the separation. A peak will appear whenever there is an analyte in a higher concentration that co-elutes with another analyte. The retention time of this peak corresponds with the identity of the higher concentration analyte and the AUC relates to the concentration proportion of analytes. The ratio chromatogram technique was utilized to extract the profile of TDNs spiked into the HS matrix. The noise threshold was set to the value associated with the maximum absorbance signal of a PBS injection. The AUC data for the ratio chromatogram analysis was normalized to the area range within each independent sample to provide the relative change in concentration of intact TDNs over time.

Statistical Analysis. All experiments were conducted in triplicate $(n=3)$. Data points on all plots are shown as the mean with error bars representing one standard deviation from the mean.

\section{RESULTS AND DISCUSSION}

Characterization of a DNA Nanostructure by SECHPLC. We selected a 17-bp edge tetrahedral DNA nanostructure (TDN) assembled by four component strands as our model system $^{14}$, as it is one of the most well-characterized DNA nanostructures used for method development in biosensing, imaging, and drug delivery applications ${ }^{15}$. To confirm the successful assembly of the TDN and map the intermediate formation products, we performed a stepwise annealing protocol through sequential equimolar addition of each of the component strands. All intermediate products were characterized by SEC-HPLC and $6 \%$ native PAGE for comparison. As shown in Figure 1a the addition of each strand results in a decrease in electrophoretic mobility of the resulting complex, leading to successively 
a)

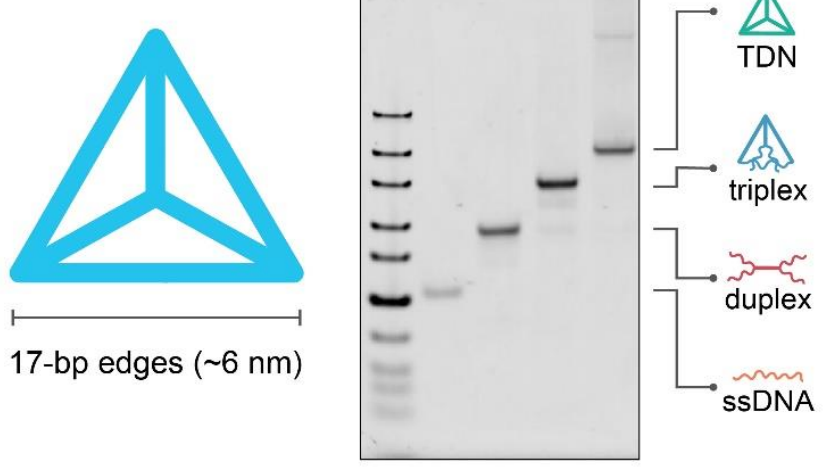

b)

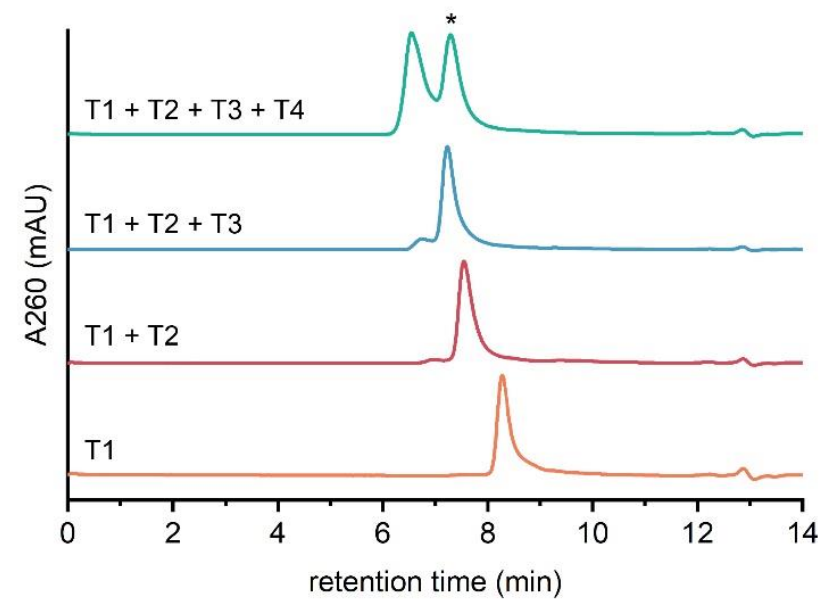

Figure 1. The successful assembly of DNA Tetrahedrons (TDNs) with edges of 17 base pairs in length is shown in (a) following a stepwise annealing procedure (Lane 1: Ultra Low-Range DNA ladder; Lane 2: T1; Lane 3: T1+T2; Lane 4: T1+T2+T3; Lane 4: T1+T2+T3+T4). (b) The samples from (a) were analyzed by SEC-HPLC to map out the range of retention times for the differently sized components in the TDN assembly. The asterisk indicates the chromatographic peak corresponding to the assembled TDNs.

higher bands on the gel. This gel mobility shift corresponds to the increase in hydrodynamic size of each complex, as evidenced by shifts to progressively earlier retention times on the SEC chromatograms ( $\underline{\text { Figure } \mathbf{1 b}})$. In an injection series, the first component strand (T1) showed a retention time of $8.277 \mathrm{~min}$, followed by $7.546 \mathrm{~min}$ for the duplex $(\mathrm{T} 1+\mathrm{T} 2), 7.229 \mathrm{~min}$ for the triplex (T1+T2+T3), and $7.289 \mathrm{~min}$ for the assembled TDN with side-product aggregates eluting at $6.550 \mathrm{~min}$. Notably, the triplex intermediate elutes at a similar retention time of the assembled TDN. This observation can be attributed to the fact that the triplex intermediate possesses a TDN-like structure with similar molecular size and rigidity ${ }^{17}$. We utilized this mapping of retention time ranges for intermediates in the TDN formation to inform our later interpretation of TDN degradation.

As the formation reaction of TDNs always results in kinetic side-products (branched chains and aggregates) ${ }^{17}$, we adapted a method to isolate purified TDNs using SEC. Following a previously-reported approach ${ }^{12}$, we used a semi-preparative SEC column in our HPLC configuration and evaluated the impact of buffer composition and flow rate on the resolution of TDNs from aggregated side-products (Figure S2). We found that the best resolution $\left(\mathrm{R}_{\mathrm{s}}=2.010\right)$ was obtained using a mobile phase of $30 \mathrm{mM}$ Tris- $\mathrm{HCl}, 450 \mathrm{mM} \mathrm{NaCl}$ at $\mathrm{pH} 7.40$ (1X TN buffer), operating at a flow rate of $0.50 \mathrm{~mL} \mathrm{~min}^{-1}$. The peak corresponding to assembled TDNs can be collected online, then concentrated to a desired final volume using 30K MWCO Amicon centrifugation filters. We loaded a maximum of $500 \mu \mathrm{L}$ of annealed TDNs $(2 \mu \mathrm{M})$ in a single injection and obtain TDNs with an average $56.7 \%$ recovery with $63.3 \%$ purity (Figure S2). Concentrated TDNs stored at $4^{\circ} \mathrm{C}$ in the purification buffer (TN) show stability consistent with previously reported observations of no apparent degradation over 30 days $^{12}$, and we used this approach to prepare TDNs prior to all stability analyses.

Our approach for the stability experiments involves incubating purified TDNs with a test matrix under relevant conditions (i.e. $37^{\circ} \mathrm{C}$ ), followed by injection onto the HPLC system at specified timepoints. We first identified the optimal set of instrument operation conditions for a complex matrix such that (1) the assembled TDNs are sufficiently resolved from aggregates and degradation products, and (2) the TDN peak elutes within the lowest background of the matrix sample - diluted human serum. We developed our stability characterization method using an analytical SEC column to reduce analysis time and minimize the amount of sample needed per injection. Samples of purified TDN and 5\% HS in phosphate-buffered saline (PBS, pH 7.40) were analyzed to evaluate the impact of buffer composition and flow rate on the separation of TDN and serum components, respectively. Our best performance was found when using PBS as the mobile phase at a $0.30 \mathrm{~mL} \mathrm{~min}^{-1}$ flow rate. The resulting chromatograms of TDN and 5\% $\mathrm{HS}$, as well as a sample of 5\% HS spiked with TDN are shown in Figure $\underline{2 a}$. The diluted HS sample chromatogram presents with three main peaks, likely corresponding to the globular protein constituents found in the highest abundance in serum samples: albumin, transferrin, and immunoglobulins, ranging in size from $(67-150 \mathrm{kDa})$. Notably, as our selected TDN has a molecular weight of $68 \mathrm{kDa}$, but a larger apparent size (>100 kDa) due to the three-dimensional geometry of the structure, the TDN peak cannot be baseline-resolved from the serum proteins due to the limited resolving power of SEC. However, under our optimized conditions, the TDN peak is eluted within the lowest background portion of the HS sample, which minimizes background signal interference upon analysis.

To calibrate the analytical performance of our method, we evaluated key metrics of injection precision as well as the limit and accuracy of TDN quantification. We prepared serial dilutions of TDN in PBS over a concentration range of $0.5-50 \mathrm{ng}$ $\mu \mathrm{L}^{-1}$ and verified the concentration by the standard measurement of the absorbance at $260 \mathrm{~nm}$ using a Nanodrop spectrophotometer. We then performed three replicate injections of each dilution and calculated the area under the curve (AUC) for the TDN peak. We generated a linear calibration curve by plotting the amount of TDN injected onto the column by the AUC values corresponding to TDN peaks, as shown in Figure $2 \boldsymbol{b}$. The limit of quantification (LOQ) of TDNs was determined to be $71.2 \mathrm{ng}$, and TDNs could be recognized by the HPLC detector at as low as $1 \mathrm{ng}$ per sample. Intra-day precision was determined for samples of TDN and 5\% HS through six sequential injections and reported in Figure $2 \boldsymbol{c}$-d . The intra-run peak retention times for both sample types were consistent within 
a)

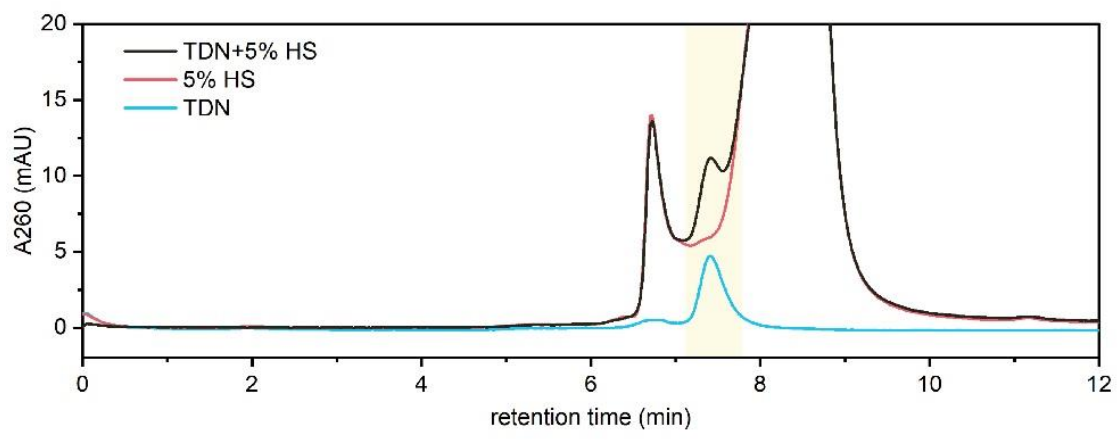

c)
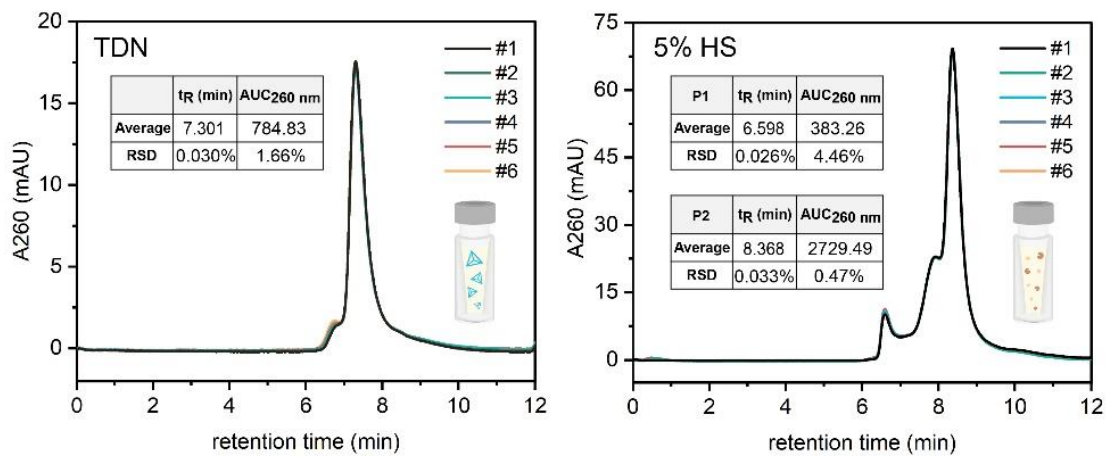

b)

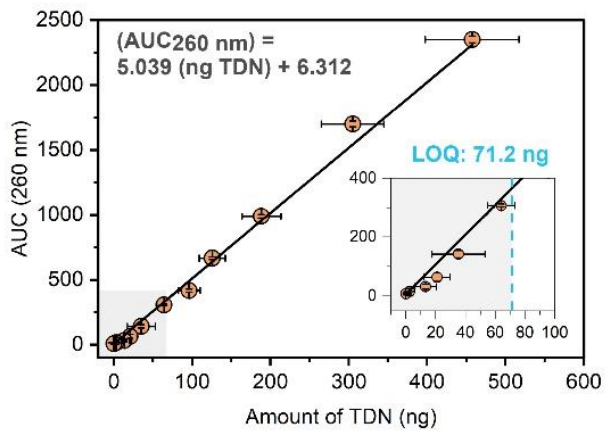

e)

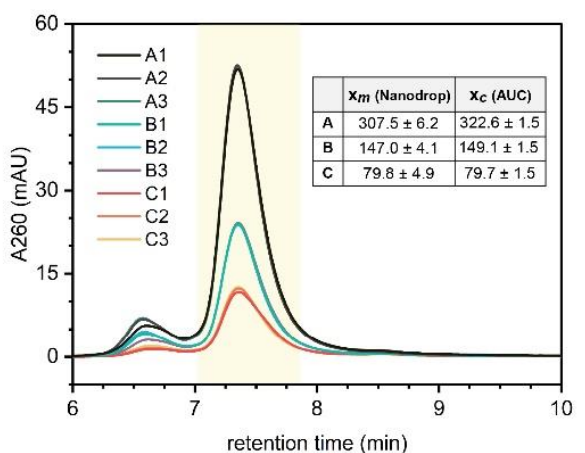

Figure 2. Analytical calibration for the SEC-HPLC method. (a) The overlayed chromatograms of TDN, 5\% HS, and TDN in 5\% HS. Running conditions of the SEC-HPLC method were optimized so that the TDN peak eluted within the lowest intensity of the human serum sample to minimize background during analysis. (b) A calibration curve of TDNs was used to determine the limit of quantification (LOQ) for TDNs at $71.2 \mathrm{ng}$. Precision analysis of replicate TDN (c) and 5\% HS (d) injections revealed minimal variations within an analysis set. Accuracy of the calibration curve was assessed in (e) using three independent TDN samples, showing less than 5\% error between experimental and theoretical quantities. The yellow highlighted region indicates the retention time range over which the TDN peak integration was determined.

$0.03 \%$ RSD, while the AUC values exhibited variation within $0.47 \%-4.46 \%$ RSD. We observed that inter-day peak retention times could vary within $\sim 0.2$ min depending on day-to-day variations in system backpressure and column performance, so the AUC integration range for stability analysis was always calibrated using a TDN-only sample in each analysis batch. Accuracy of the calibration method was probed using independent samples of TDN at three different concentrations across the calibration range, with measurement by Nanodrop and our SEC method. The resulting data is shown in Figure 2e, where the quantification measurements were found to be accurate within $0.05 \%$ RSD towards the lower end of the curve $(\sim 80 \mathrm{ng})$ and within $4.94 \%$ RSD towards the higher end ( $300 \mathrm{ng})$.

Probing the Degradation of a TDN by Direct-Injection. We conducted a time-lapse stability analysis using the SEC method conditions adjusted and calibrated for the analyte. To first understand the degradation profile of TDNs without interference from the HS matrix components, we incubated DNaseI with our sample. DNaseI is a non-specific endonuclease and the most abundant nuclease found in human serum ${ }^{20}$. This nuclease is typically used in characterization studies of DNA nanostructures, particularly for assessing the biostability improvement of various stabilization strategies ${ }^{1}$. The average range for DNaseI activity in the blood plasma of healthy donors is from $0.1-0.9$ $\mathrm{U} \mathrm{mL}^{-1}$ but it can vary by as much as $0.004-6 \mathrm{U} \mathrm{mL}^{-1}$ in response to various illnesses ${ }^{21}$. We analyzed TDNs incubated at $37^{\circ} \mathrm{C}$ with DNaseI at a concentration of $1.56 \mathrm{U} \mathrm{mL}^{-1}$, which was chosen to maximize the number of data points that could be acquired at $\sim 15 \mathrm{~min}$ intervals - the length of one full chromatographic separation - while minimizing the total analysis time. The mixed sample was prepared and incubated in an autosampler vial, then moved to directly inject onto the HPLC during the specified time intervals. As shown in Figure 3a, the TDN peak at $\sim 7.3$ min was visibly degraded over the course of the incubation, while the side-product peak at $\sim 6.8$ min remained largely undigested. We hypothesize that this may be a result of the branched side-product aggregates containing numerous single-stranded domains ${ }^{17}$, and DNaseI cleaves dsDNA domains 100-500 times more effectively than ssDNA ${ }^{20}$. The presence of increased absorbance of irregular peaks in the $\sim 8$ 11 min retention time range for the later timepoints corresponds to the presence of short oligonucleotide degradation products. A sample of DNaseI and its reaction buffer components at the same concentration used in this experiment did not show any visible peaks on the chromatogram, so interpretation of this data was treated as "matrix free". The plot of averaged AUC values for the $\mathrm{A}_{260}$ chromatograms over time for three digestion replicate experiments is shown in Figure $\mathbf{3 b}$. A first-order exponential decay curve fit to the data results in a calculated half-life of $11.63 \mathrm{~min}$. This is consistent with previously reported stability data for this TDN design, where it was fully degraded in $10 \mathrm{~min}$ at a $10 \mathrm{U} \mathrm{mL}^{-1} \mathrm{DNaseI}$ concentration (approximate half-life of less than $2 \mathrm{~min})^{16}$. 
a)

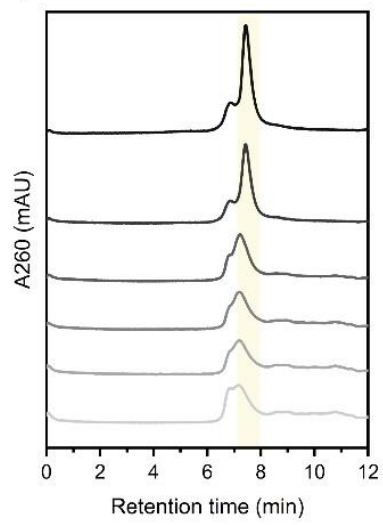

b)

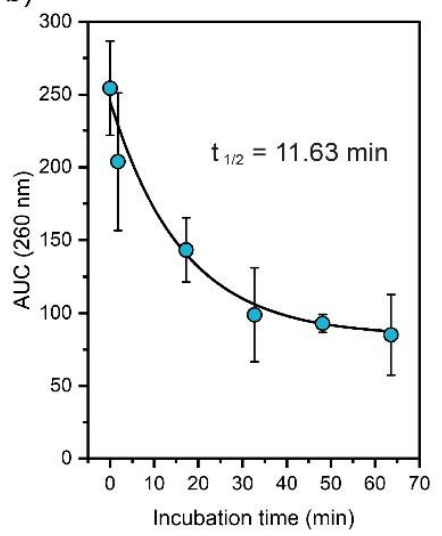

Figure 3. DNaseI digestion assay. (a) An example of the TDN chromatograms overlayed for one replicate of the DNaseI digestion at time points of $0.0,1.8,17.2,32.7,48.1$, and $63.6 \mathrm{~min}$. The yellow highlighted region indicates the retention time range over which the TDN peak integration was determined. Notably, the TDN peak diminished over the incubation while the aggregate peak remained after one hour. We attribute this likely to the aggregate side products possessing numerous ssDNA domains; DNaseI digests dsDNA at a rate 100-500 times that of ssDNA. (b) The integrated TDN peak area over time follows a decay trend with a half-life of $11.63 \mathrm{~min}$, which is consistent with reported values.

Characterizing the Stability of a TDN in HS by Ratiometric Analysis. The DNaseI digestion study revealed that our SEC approach can produce stability information that is consistent with the standard assays that use gel electrophoresis. However, the direct-injection approach used for the enzymatic assay would not be feasible with undiluted human serum (HS) as it contains a high protein content $\left(\sim 70 \mu \mathrm{g} \mathrm{L} \mathrm{L}^{-1}\right)$, which would overload the analytical HPLC system and cause persistent clogging. We evaluated the effect of various concentrations of HS diluted in PBS and observed that clogging consistently occurs at sample injection concentrations of $10 \%$ HS or higher. We found that injections at an effective concentration of 5\% $\mathrm{HS}$ $\left(\sim 3-4 \mu \mathrm{g} \mu \mathrm{L}^{-1}\right.$ protein) result in sufficient signal for analysis above our detection limits, without clogging the system. However, if we incubated TDNs in a 5\% HS matrix, the slower halflife of degradation would lead to significantly longer processing times for each experiment. We prepared TDN samples mixed with diluted HS in PBS at concentrations of $20 \%$ and $50 \%$, then diluted an aliquot of each sample to an effective concentration of 5\% HS prior to injection. These concentrations were chosen to balance a gradual decay profile of the TDNs to enable fitting to a decay curve while minimizing the length of time to complete the workflow. Dilution of the sample before injection induces additional error into the data, so directly measuring the AUC of the $\mathrm{A}_{260}$ chromatogram would not be reliable for quantitative assessment. As such, we adapted a ratiometric approach inspired by Synovec et al ${ }^{18}$ by plotting the detected $\mathrm{A}_{260}$ by $\mathrm{A}_{280}$ over the course of the separation as a ratio chromatogram. Further details of this ratiometric analysis technique are outlined in the Experimental Section. One of the key advantages of using an SEC-HPLC method for stability analysis is the ability to simultaneously monitor the sample absorbance at multiple different wavelengths or even collect an entire spectrum at each point in the analysis. The 260/280 ratio is widely accepted as a metric for assessment of the purity of DNA and protein samples, and it is highly influenced by DNA contamination in protein samples. Here, we exploit this optical phenomenon to deconvolute our TDN peak from the co-eluting matrix of serum proteins. In Figure 4a, the chromatograms for TDN, HS, and TDN in HS are displayed, with the highlighted region showing the range for integration. This example data is converted to 260/280 ratio chromatograms in Figure $\mathbf{4 b}$. Over pure-elution regions in each profile, the plot simplifies to a plateau with a value consistent with the identity of the analyte. The TDN elution region shows a value of $\sim 1.8$, consistent with that of a pure DNA sample. The profile for HS exhibits flat regions with values ranging from $0.53-0.60$, consistent with protein elution, changing in value when the serum protein components change. A peak at $\sim 7.4 \mathrm{~min}$ appears in the spiked sample, consistent with TDN co-eluting in a higher concentration proportion to the serum proteins.

To evaluate the time-resolved stability of TDN in HS, we prepared samples of TDN ( 1000 ng) spiked into 20\% and 50\% HS in PBS, incubated at $37^{\circ} \mathrm{C}$. Before HPLC injection, an aliquot of the test sample was diluted to an effective 5\% HS concentration (e.g. 1:4 for 20\% HS, 1:10 for 50\% HS) and injected onto the column at designated time points. The AUC over the range for the intact TDN was calculated using the ratio chromatograms and plotted by incubation time as shown in Figure 4c$f$. By assuming the degradation process follows first-order exponential decay, we calculated half-lives of $23.91 \pm 1.23 \mathrm{hrs}$ for TDN in 20\% HS and $10.14 \pm 0.77 \mathrm{hrs}$ for TDN in 50\% HS. By extrapolating this information, we estimate that the half-life in this whole human serum sample would be $\sim 4.5-5.5 \mathrm{hrs}$. Independent samples of 5\% HS and TDN were also analyzed through incubation at $37^{\circ} \mathrm{C}$ (Figure S4). The samples containing 5\% HS showed minimal degradation changes while the sample of TDN in PBS exhibited only a 20\% loss of intact structures over the 70-hr period. We note that as the nuclease activity in human sera varies greatly depending on the donor's health status, the stability of TDNs in serum samples from different donor sources is likely to vary. However, our approach can provide a good approximation of expected degradation, and a useful means to compare the degradation profiles of various DNA nanostructure designs in the development phase. Our stability data is consistent with previously-reported metrics where this 17-bp edge TDN was fully degraded in $42 \mathrm{~h}$ in $10 \%$ fetal bovine serum $(\mathrm{FBS})^{16}$, which has greater than $0.256 \mathrm{U} \mathrm{mL}^{-1}$ DNase activity $^{4}$. To demonstrate the limitations of the gel electrophoresis method for characterizing DN stability in HS, we prepared samples of TDN spiked into 5-50\% HS over ice to minimize degradation, and analyzed the samples by $6 \%$ native PAGE, shown in Figure S5. As seen on the gel, background signal from serum proteins starts to interfere with the intensity of the TDN band on the gel at 5\% HS and as the concentration increases, the TDN band apparently disappears due to aggregate interaction with HS proteins. Additionally, background signal from the DNA stain interacting with the proteins complicates the analysis. In contrast, our SEC method obtained this native stability information without requiring purification or extraction of the DNA prior to analysis, as is needed when using the traditional gel electrophoresis assay for assessing stability in biofluids. 

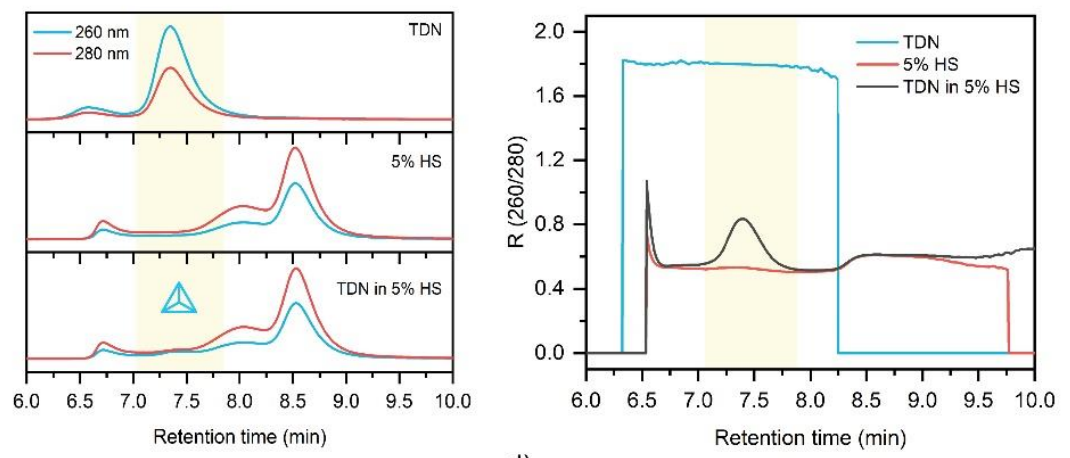

c)

d)
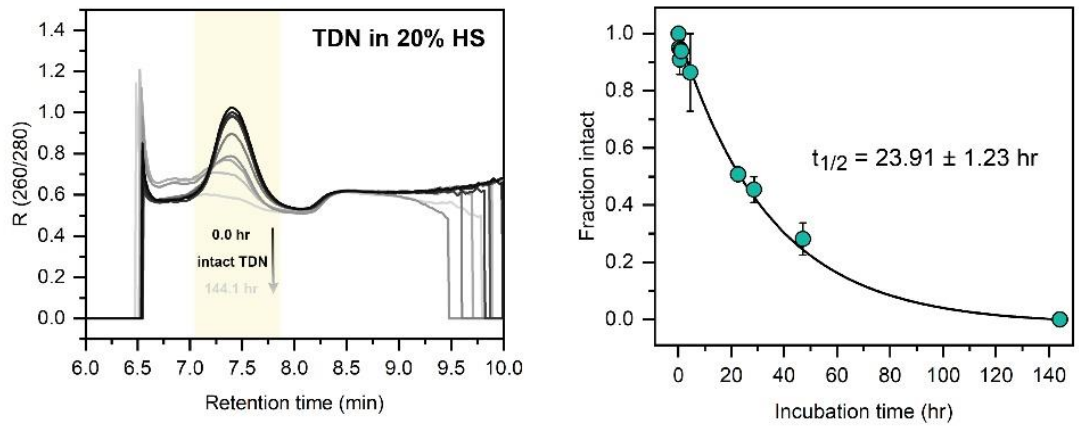

e)

f)
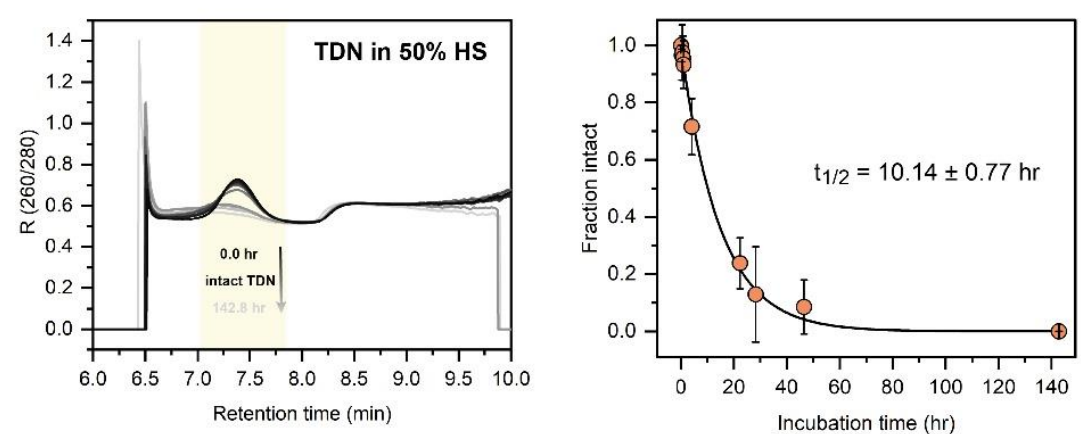

Figure 4. Serum stability analysis of a TDN by a ratiometric method. (a) The chromatographic profiles of samples of TDN, 5\% HS, and TDN in 5\% HS at $260 \mathrm{~nm}$ and $280 \mathrm{~nm}$ are displayed for comparison. The yellow highlighted region indicates the retention time range over which the TDN peak integration was determined. The data from (a) is converted into an overlayed ratio chromatogram as shown in (b). Notably, the TDN ratio profile simplifies to a flat region with a value of $\sim 1.8$, consistent with that of a pure DNA sample. The profile for HS possesses flat regions with values ranging from $0.53-0.60$, consistent with protein elution, changing in value when the serum protein components change. A peak at 7.4 min appears in the spiked sample, consistent with TDN co-eluting in a higher concentration proportion to the serum proteins. Ratio chromatogram time overlays are plotted for TDN stability analysis in $20 \%$ HS (c) and 50\% HS (e). The normalized AUC for each replicate was plotted in (d) and (f), with first-order exponential decay fitting. The half-lives for the TDN were found to be $23.91 \pm 1.23 \mathrm{hr}$ and $10.14 \pm 0.77 \mathrm{hr}$ for $20 \% \mathrm{HS}$ and $50 \% \mathrm{HS}$ samples, respectively.

\section{CONCLUSIONS}

In this work, we demonstrate that the stability of a DNA nanostructure can be well-characterized using a simple SECHPLC approach. With a ratiometric data analysis technique, the TDN peak can be deconvoluted from an HS matrix in order to examine the native stability in a complex biological environment. This technique has the potential to enable comparative assay of a wide variety of DNs. We suggest that future work could explore the use of coupling an SEC-HPLC analysis with other downstream analytical techniques.

\section{ASSOCIATED CONTENT}

\section{Supporting Information}

DNA sequences, additional figures, and data analysis methods $(P D F)$

\section{Conflicts of Interest}

There are no conflicts to declare. 


\section{AUTHOR INFORMATION}

* Heather A. Clark - Northeastern University, Boston, Massachusetts; orcid.org/0000-0002-2628-9194; Email: h.clark@ northeastern.edu (*Corresponding author)

Nicole I. Langlois - Northeastern University, Boston, Massachusetts; orcid.org/0000-0002-2530-9739; Email: langlois.n@ northeastern.edu

\section{ACKNOWLEDGMENTS}

This work was supported through the National Institutes of Health, National Institute of Biomedical imaging and Bioengineering through award number 3R01EB024186-03S1. The authors would like to thank Taylor Lynch for assistance in carrying out preliminary studies and Isen Calderon for helpful discussions regarding experimental design and data analysis.

\section{REFERENCES}

1. Chandrasekaran, A. R.; Halvorsen, K., Nuclease Degradation Analysis of DNA Nanostructures Using Gel Electrophoresis. 2020, 82 (1), e115.

2. Keum, J.-W.; Bermudez, H., Enhanced resistance of DNA nanostructures to enzymatic digestion. Chemical Communications 2009, (45), 7036-7038.

3. Conway, J. W.; McLaughlin, C. K.; Castor, K. J.; Sleiman, H., DNA nanostructure serum stability: greater than the sum of its parts. Chemical Communications 2013, 49 (12), 1172-1174.

4. Hahn, J.; Wickham, S. F. J.; Shih, W. M.; Perrault, S. D., Addressing the instability of DNA nanostructures in tissue culture. ACS Nano 2014, 8 (9), 8765-8775.

5. Perrault, S. D.; Shih, W. M., Virus-Inspired Membrane Encapsulation of DNA Nanostructures To Achieve In Vivo Stability. ACS Nano 2014, 8 (5), 5132-5140.

6. $\quad$ Goltry, S.; Hallstrom, N.; Clark, T.; Kuang, W.; Lee, J.; Jorcyk, C.; Knowlton, W. B.; Yurke, B.; Hughes, W. L.; Graugnard, E., DNA topology influences molecular machine lifetime in human serum. Nanoscale 2015, 7 (23), 10382-10390.

7. Chandrasekaran, A. R., Nuclease resistance of DNA nanostructures. Nature Reviews Chemistry 2021, 5 (4), 225-239.

8. Lacroix, A.; Vengut-Climent, E.; de Rochambeau, D.; Sleiman, H. F., Uptake and Fate of Fluorescently Labeled DNA Nanostructures in Cellular Environments: A Cautionary Tale. ACS Central Science 2019, 5 (5), 882-891.

9. Mathur, D.; Medintz, I. L., Analyzing DNA nanotechnology: a call to arms for the analytical chemistry community. Analytical Chemistry 2017.
10. Striegel, A.; Yau, W. W.; Kirkland, J. J.; Bly, D. D., Modern size-exclusion liquid chromatography: practice of gel permeation and gel filtration chromatography. John Wiley \& Sons: 2009.

11. Hong, P.; Koza, S.; Bouvier, E. S. P., A Review SizeExclusion Chromatography for the Analysis of Protein Biotherapeutics and Their Aggregates. Journal of Liquid Chromatography \& Related Technologies 2012, 35 (20), 2923-2950.

12. Xing, S.; Jiang, D.; Li, F.; Li, J.; Li, Q.; Huang, Q.; Guo, L.; Xia, J.; Shi, J.; Fan, C.; Zhang, L.; Wang, L., Constructing HigherOrder DNA Nanoarchitectures with Highly Purified DNA Nanocages. ACS Applied Materials \& Interfaces 2015, 7 (24), 13174-13179.

13. Shaw, A.; Benson, E.; Högberg, B. r., Purification of functionalized DNA origami nanostructures. ACS Nano 2015, 9 (5), 4968-4975.

14. Goodman, R. P.; Berry, R. M.; Turberfield, A. J., The single-step synthesis of a DNA tetrahedron. Chemical Communications 2004, (12), 1372-1373.

15. Xie, N.; Liu, S.; Yang, X.; He, X.; Huang, J.; Wang, K., DNA tetrahedron nanostructures for biological applications: biosensors and drug delivery. Analyst 2017, 142 (18), 3322-3332.

16. Keum, J.-W.; Bermudez, H., Enhanced resistance of DNA nanostructures to enzymatic digestion. Chemical Communications 2009, 7 (45), 7036-7038.

17. Gao, L.; Liu, L.; Tian, Y.; Yang, Q.; Wu, P.; Fan, C.; Zhao, Q.; Li, F., Probing the Formation Kinetics and Thermodynamics with Rationally Designed Analytical Tools Enables One-Pot Synthesis and Purification of a Tetrahedral DNA Nanostructure. Analytical Chemistry 2021, 93 (18), 7045-7053.

18. Synovec, R. E.; Johnson, E. L.; Bahowick, T. J.; Sulya, A. W., Ratio of sequential chromatograms for quantitative analysis and peak deconvolution: application to standard addition method and process monitoring. Analytical Chemistry 1990, 62 (15), 1597-1603.

19. Bahowick, T. J.; Dunphy, D. R.; Synovec, R. E., Analysis of unresolved chromatograms by the absorbance ratio and sequential chromatogram ratio techniques coupled with peak suppression. Journal of Chromatography A 1994, 663 (2), 135-150.

20. Lauková, L.; Konečná, B.; Janovičová, L.; Vlková, B.; Celec, P., Deoxyribonucleases and Their Applications in Biomedicine. Biomolecules 2020, 10 (7).

21. Tamkovich, S. N.; Cherepanova, A. V.; Kolesnikova, E. V.; Rykova, E. Y.; Pyshnyi, D. V.; Vlassov, V. V.; Laktionov, P. P., Circulating DNA and DNase Activity in Human Blood. 2006, 1075 (1), 191-196.

Table of Contents Figure:

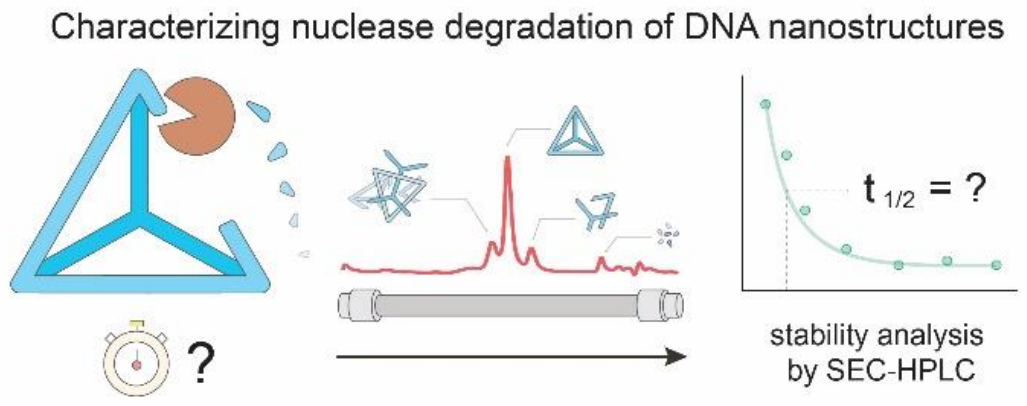

